\title{
Worenine reverses the Warburg effect and inhibits colon cancer cell growth by negatively regulating HIF-1a
}

\author{
Lijiang Ji1,2†, Weixing Shen ${ }^{2,4 \dagger}$, Feng Zhang ${ }^{2}$, Jie Qian², Jie Jiang ${ }^{1,2}$, Liping Weng ${ }^{1}$, Jiani Tan ${ }^{2,4}$, Liu Li ${ }^{2,4}$, \\ Yugen Chen ${ }^{2,4}$, Haibo Cheng ${ }^{2,4^{*}}$ and Dongdong Sun ${ }^{2,3^{*}}$
}

${ }^{*}$ Correspondence: hbcheng_njucm@163.com; sundd@njucm.edu.cn ${ }^{\dagger} \mathrm{Lijiang} \mathrm{Ji}$ and Weixing Shen have contributed equally to this work.

2 Collaborative Innovation Center of Jiangsu Province of Cancer Prevention and Treatment of Chinese Medicine, Nanjing 210023,

China

Full list of author information is available at the end of the article

\begin{abstract}
Background: Some natural compounds inhibit cancer cell growth in various cancer cell lines with fewer side effects than traditional chemotherapy. Here, we explore the pharmacological effects and mechanisms of worenine (isolated from Coptis chinensis) against colorectal cancer.

Methods: The effects of worenine on colorectal cancer cell proliferation, colony formation and cell cycle distribution were measured. Glycolysis was investigated by examining glucose uptake and consumption, lactate production, and the activities and expressions of glycolysis enzymes (PFK-L, HK2 and PKM2). HIF-1a was knocked down and stimulated in vitro to investigate the underlying mechanisms.

Results: Worenine somewhat altered the glucose metabolism and glycolysis (Warburg effect) of cancer cells. Its anti-cancer effects and capability to reverse the Warburg effect were similar to those of HIF-1a siRNA and weakened by deferoxamine (an HIF-1a agonist).
\end{abstract}

Conclusion: It is suggested that worenine targets HIF-1a to inhibit colorectal cancer cell growth, proliferation, cell cycle progression and the Warburg effect.

Keywords: Worenine, Warburg effect, Colon cancer, HIF-1a, Glycolysis

\section{Background}

Colorectal cancer is the third most common cancer and the fourth leading cause of cancer-associated death globally $[1,2]$. Conventional chemotherapy has shown no significant benefit in improving overall survival rates and it has proven to increase patients' chronic pain. Surgery is combined with chemotherapy has been employed clinically, but the results were far from satisfactory [3]. Therefore, it is essential to develop novel anticolorectal cancer agents with lower toxic and greater efficacy.

The dried rhizome of Coptis chinensis (Chinese goldthread, family Ranunculaceae) has been reported to possess anti-tumor activities in vitro and in vivo $[4,5]$. It contains a range of bioactive ingredients, including ferulic acid, berberine, palmatine, magnoflorine, coptisine and worenine [6]. Coptisine showed anti-tumor activities 
against colon cancer through the induction of apoptosis without toxic symptoms [7]. Worenine is an isoquinoline alkaloid that has a similar chemical structure to coptisine (Fig. 1a) [8]. Therefore, we hypothesized that worenine might be a promising low toxicity compound for colorectal cancer treatment.

Unlike normal cells, most cancer cells exhibit an energy metabolism disorder that contributes to their growth and proliferation. They also display the Warburg effect: active glucose uptake and glycolysis under aerobic conditions [9]. Although the mechanisms of the Warburg effect have not been fully elucidated, multiple factors are

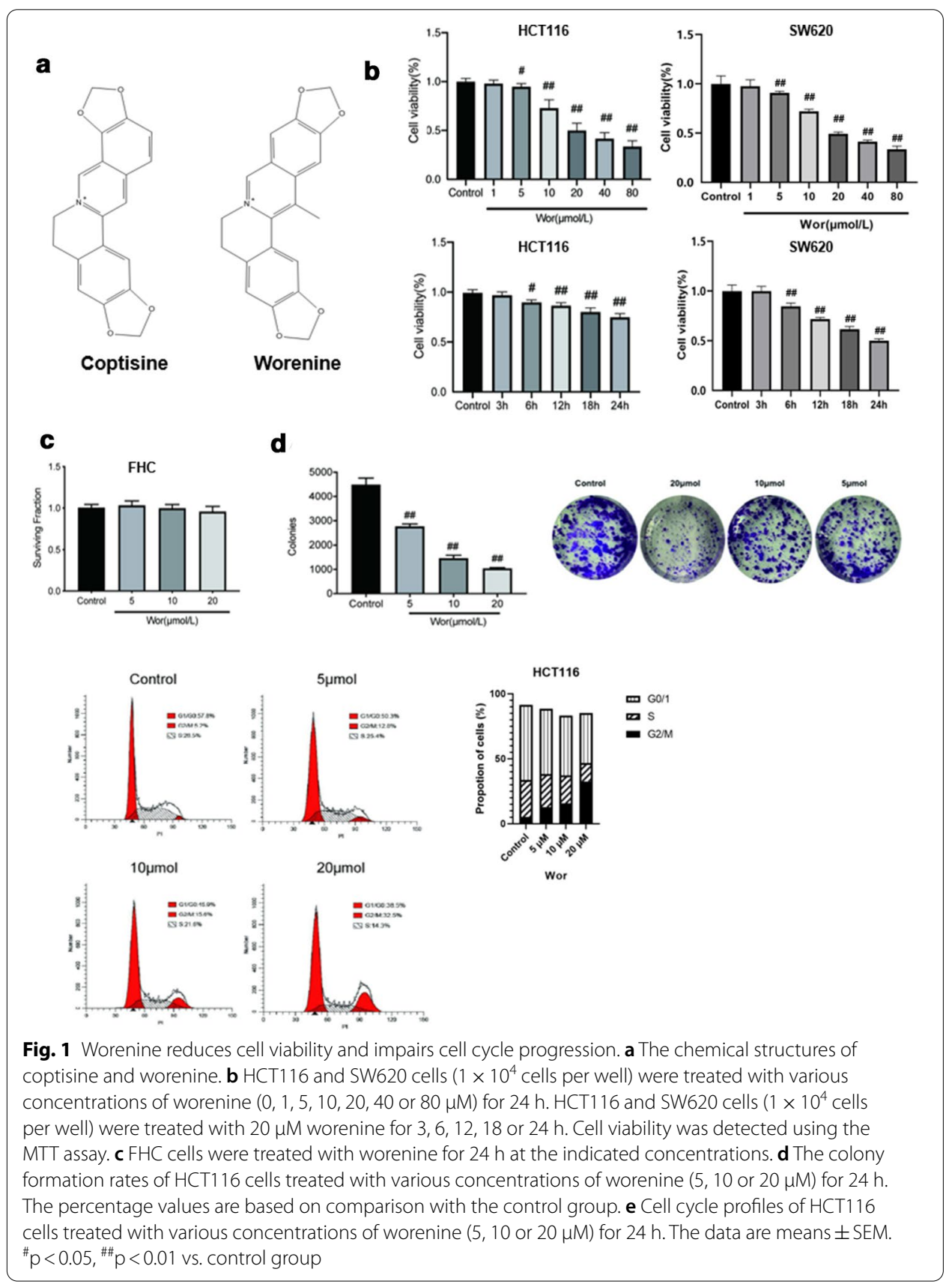


known to regulate it, including mitochondrial oxidative phosphorylation, glycolysis enzymes, oncogenes, signal transduction pathways and the tumor microenvironment.

Hypoxia-inducible factor $1 \alpha$ (HIF-1 $\alpha)$ is a key mediator of the Warburg effect in tumor cells. It activates the transcription of genes that encode proteins in oxygen-independent energy production pathways. It can promote cell glycolysis in the tumor microenvironment under hypoxic conditions by upregulating the expression of glycolytic enzymes, such as glucose transporters 3 (GLUT3), phosphofructokinase 1 (PFK1), hexokinase 2 (HK2), lactate dehydrogenase A (LDHA) and pyruvate kinase M2 (PKM2) [10].

Here, we assessed the effect of worenine on cell proliferation, cell cycle distribution and colony formation in colorectal cancer. Then we investigated glycolysis by measuring glucose uptake and consumption, lactate production, and the expressions of the glycolysis enzymes GLUT3, LDHA, PFK-L, HK2 and PKM2. We found that worenine altered the Warburg effect in the cancer cells. Using HIF-1 $\alpha$ knockdown and stabilization, we demonstrated that the anti-colorectal cancer effects of worenine are associated with HIF-1 $\alpha$-modulated glycolysis. This is the first investigation of the in vitro regulatory impact of worenine on the Warburg effect in colorectal cancer.

\section{Materials and methods}

\section{Cell culture}

Worenine with a purity of at least $97 \%$ was obtained from Shanghai Yuanye Bio-Technology and dissolved in dimethyl sulfoxide (DMSO). The colorectal cancer cell lines HCT116 and SW620 and the normal human colon cell line FHC (CRL-1831) were purchased from the cell bank of the Chinese Academy of Sciences (CAS). The cancer cell lines were cultured in RPMI 1640 medium (Biological Industries, Israel) containing 10\% $(\mathrm{v} / \mathrm{v})$ fetal bovine serum (FBS; Thermo Fisher Scientific, USA) and 100 units per ml of streptomycin and penicillin (Sigma, USA). FHC cells were cultured in DMEM/F12 (Dulbecco's modified Eagle medium; Gibco, New Zealand) containing 10\% FBS, 2.5\% L-Glutamine and 100 units per $\mathrm{ml}$ of penicillin and streptomycin. All cells were incubated in a humidified incubator at $37^{\circ} \mathrm{C}$ with $5 \% \mathrm{CO}_{2}$.

\section{MTT test}

The effect of worenine treatment on the cell viability of HCT-116 cells was determined using a Beyotime MTT test kit (C0009) according to the manufacturer's instructions. Cells were seeded into 96-well plates and cultured for $24 \mathrm{~h}$, followed by $24 \mathrm{~h}$ of worenine treatment. Then, $20 \mu \mathrm{l} /$ well of MTT $(5.0 \mathrm{mg} / \mathrm{ml})$ was added and cultured for $4 \mathrm{~h}$. The cell supernatant was removed and replenished with $200 \mu \mathrm{l}$ DMSO to dissolve the formazan precipitate. The UV absorption at $490 \mathrm{~nm}$ was measured using a microplate reader (Elx800, BioTek, USA).

\section{Colony formation test}

A colony formation test was performed to further assess the effect of worenine on cell viability. HCT-116 cells were seeded into 6-well dishes and incubated for $24 \mathrm{~h}$. Afterwards, cells were treated with 5, 10 and $20 \mu \mathrm{M}$ worenine for $24 \mathrm{~h}$. The cell culture was substituted with fresh medium every 2 days. After 2 weeks, colonies were fixed 
in methanol and stained with $0.1 \%$ crystal violet. Colonies with at least 50 cells were counted under an inverted microscope.

\section{Cell cycle analysis}

Propidium iodide (PI) RNase staining solution (4087S, CST) was used for the cell cycle assay. HCT-116 cells were incubated with worenine for $24 \mathrm{~h}$. Afterwards, the cells were washed once with phosphate-buffered saline (PBS) and fixed overnight with $500 \mu \mathrm{l}$ chilled $70 \%$ ethanol at $4{ }^{\circ} \mathrm{C}$, then washed again with PBS. They were then suspended with the staining solution as described previously and incubated in darkness at $4{ }^{\circ} \mathrm{C}$ for 30 min. Finally, the cells were quantified using FACS-Calibur flow cytometry (BD Biosciences, USA).

\section{Assay of the glucose uptake and consumption levels and lactate production level}

After worenine treatment for $72 \mathrm{~h}$, the medium was collected for lactate and glucose concentration analysis. The lactate content in the media were tested according to the manufacturer's instructions for the Lactic Acid Production Detection kit (Nanjing Jiancheng Bioengineering Institute, China). To evaluate the glucose uptake and consumption, a Glucose Uptake Colorimetric Assay Kit (Sigma, USA) and a Glucose Assay Kit (Solarbio Science \& Technology, China) were respectively used according to the manufacturers' instructions.

\section{Western blot analysis}

Cells were rinsed three times with ice-cold PBS, lysed in RIPA buffer for $30 \mathrm{~min}$ at $4{ }^{\circ} \mathrm{C}$, and then centrifugated at $8000 \mathrm{~g}$ for $10 \mathrm{~min}$ at $4{ }^{\circ} \mathrm{C}$. The protein concentration was measured using the Bradford protein assay. The membrane and nuclear proteins of the HCT116 cell were extracted and isolated using a Cell membrane-Cytoplasm-Nucleoprotein Stepwise Extraction Kit (BB31042, Bestbio). Using sodium dodecylsulphatepolyacrylamide gel electrophoresis (SDS-PAGE), $50 \mu \mathrm{g}$ of protein was purified and then transferred to polyvinylidene difluoride (PVDF) membrane. Following blocking with 5\% non-fat milk (0.05\% Tween 20 Tris-saline) at room temperature for $2 \mathrm{~h}$, the membranes were incubated with primary antibodies (Abcam, USA) against HIF-1 $\alpha$ (1:1000), p-VHL (1:1000), $\beta$-actin (1:5000), lamin B1 (1:1000), Na/K-ATPase (1:1000), PFK-L (1:10,000), HK-2 (1:1000), PKM-2 (1:1000), GLUT3 (1:1000) and LDHA (1:1000) at $4{ }^{\circ} \mathrm{C}$ overnight. The membranes were then incubated with secondary antibodies (1:10,000; Proteintech Group, USA), then washed three times with TBST. Bands were visualized with an enhanced chemiluminescence system (Pierce Biotechnology) and measured using ImageJ software (National Institutes of Health). Each of the experiments was independently repeated three times.

\section{RT-PCR analysis}

The expression levels of PFK-L and HK-2 mRNA in cells were detected using RT-PCR using our laboratory protocol [11]. Briefly, total RNA was extracted using Trizol (Invitrogen, USA), according to the manufacturer's instructions. RT-PCR was performed using the StepOne Real- Time PCR system (Applied Biosystems, USA), and the $2^{-\Delta \Delta \mathrm{Ct}}$ 
method was utilized to calculate the relative expressions of the targets. $\beta$-actin served as the internal control.

The primer sequences were designed by Sangon Biotech (China) and were: HK-2 forward, 5'-CGACAGCATCATTGTTAAGGAG-3' and reverse, 5'-GCAGGAAAGACA CATCACATTT-3'; and PKM2 forward, 5'-CCTCCTTCAAGTGCTGCAGT- $3^{\prime}$ and reverse, $5^{\prime}$-TCATGGCAAAGTTCACCCGG-3'. The experiments were repeated in triplicate.

\section{Immunofluorescence assay}

After treatment with worenine, cells were fixed in 4\% paraformaldehyde for 15 min, washed 3 times in PBS and blocked with 5\% BSA for the indicated times. Next, the prepared cells were dyed at $4{ }^{\circ} \mathrm{C}$ with anti-GAPDH (1:50), anti-PFKL (1:100) and anti-HK-2 (1:100) overnight. After being rinsed 3 times in PBS, the cells were incubated with FITClabeled secondary antibodies $(1: 100)$ for $1 \mathrm{~h}$ at $37^{\circ} \mathrm{C}$ in the dark. To label the nuclei, cells were counter-stained with DAPI (Beyotime Institute of Biotechnology, China). Images were acquired using a fluorescent microscope (Leika, Germany). The results were obtained from triplicate experiments.

\section{ELISA assay}

The concentrations of protein were evaluated using the Human Hexokinase 2 (HK-2) ELISA Kit, Human Phosphofructokinase L (PFK-L) ELISA Kit, and Human Pyruvate Kinase Isozymes M2 (PKM2) ELISA Kit (Bio-Swamp, China). The samples for ELISA were prepared as follows: the treated HCT-116 cells were collected and centrifuged at $8000 \mathrm{~g}$ for $15 \mathrm{~min}$ at $4{ }^{\circ} \mathrm{C}$ and the cell supernatant was stored at $-20^{\circ} \mathrm{C}$. The absorbance of all the samples was measured using an ELISA reader (Bio-Tek, USA), and the data were processed using Bio-Tek software.

\section{RNAi treatments}

siRNA targeting HIF- $1 \alpha$ was purchased from Burson-Marstor Bioengineering (China). The antisense sequence of the siRNA against HIF- $1 \alpha$ is 5 -AGTTCACCTGAGCCT AATA-3. The HIF-1 $\alpha$ siRNA (100 nM) was transfected into HCT116 cells using Lipofectamine 2000 according to the manufacturer's instructions (Invitrogen Life Technologies, USA).

\section{Enzyme activity analysis}

The hexokinase (HK) activity was quantified using a hexokinase activity assay kit (BC0740; Solarbio). The phosphofructokinase (PFK) and pyruvate kinase (PKM) activities were respectively measured with a phosphofructokinase activity detection kit (BC0535; Solarbio) and pyruvate kinase assay kit (ab83432, Abcam). Generally, cells $\left(5 \times 10^{6}\right)$ in $1 \mathrm{ml}$ of extracting solution were broken using ultrasonication on ice and then centrifuged at $8000 \mathrm{~g}, 4{ }^{\circ} \mathrm{C}$, for $10 \mathrm{~min}$. The supernatant was measured spectrophotometrically at $340 \mathrm{~nm}$. The HK, PFK and PKM2 activities were calculated based on the protein concentration in the sample using a BCA assay (Beyotime, China). 


\section{Statistical analysis of data}

Statistical analysis was done with SPSS 20.0 software. All data are expressed as the means \pm SEM and were obtained from multiple independent experiments. One-way ANOVA was used to detect differences among different groups and $\mathrm{p}<0.05$ was considered significant.

\section{Results}

\section{Worenine inhibits colorectal cancer cell viability and causes cell cycle arrest}

The MTT assay was used to examine the effect of worenine on two human colorectal cancer cell lines, HCT116 and SW620. Worenine was found to repress colorectal cancer cell viability at concentrations ranging from 5 to $20 \mu \mathrm{M}$ (Fig. 1b). The $\mathrm{IC}_{50}$ for HCT116 and SW620 cells were 18.30 and $15.19 \mu \mathrm{M}$, respectively. Worenine also affected the cell viability of colorectal cancer cells in a time-dependent manner. Importantly, it was shown to have anticancer effects without causing significant cell death of normal FHC human colon cells (Fig. 1c), indicating that worenine is likely to be a safe anti-colorectal cancer compound.

A cell colony formation experiment was used to further explore the long-term anticolorectal cancer effects of worenine. The number of cell colonies decreased with increasing worenine doses (Fig. 1d, Additional file 1: Figure S1A).

In addition, worenine triggered cell cycle arrest and increased the G2-to-M cell ratio (enhanced the G2/M peak; Fig. 1e, Additional file 1: Figure S1B). These results reveal that worenine can inhibit cell viability and induce cell cycle arrest in colorectal cancer cells in vitro.

\section{Worenine significantly dampens glycolysis in HCT116 cells}

To explore the effect of worenine on colorectal cancer cell glycolysis, we measured worenine-induced changes in the levels of lactic acid production as well as glucose uptake and consumption. In both HCT116 and SW620 cells, worenine treatment significantly suppressed lactic acid production and glucose uptake and consumption at concentrations of $10 \mu \mathrm{M}$ and $20 \mu \mathrm{M}$. (Fig. 2a-c). These results indicated that the Warburg effect of colorectal cancer cells is weakened by worenine. However, worenine had no significant effect on the glycolysis of FHC cells compared with the control cells (Additional file 2: Figure S2).

\section{Worenine inhibits key enzymes in glycolysis}

We further investigated the mechanism by which worenine inhibits glycolysis by examining several key glycolytic enzymes that are overexpressed in colorectal cancer cells: GLUT3, HK-2, PFK-L, PKM2 and LDHA. High doses of worenine significantly decreased the protein and mRNA levels of GLUT3, HK-2, PFK-L, PKM2 and LDHA (Fig. 3a-c, Additional file 3: Figure S3A andB). The enzyme activities of PFK, HK, PKM were also measured (Additional file 3: Figure S3C). The results indicate that the activity of PKM in HCT116 cells decreased with increasing worenine concentration. The activity of HK went up slightly when treated with worenine. This may be due to the slight 

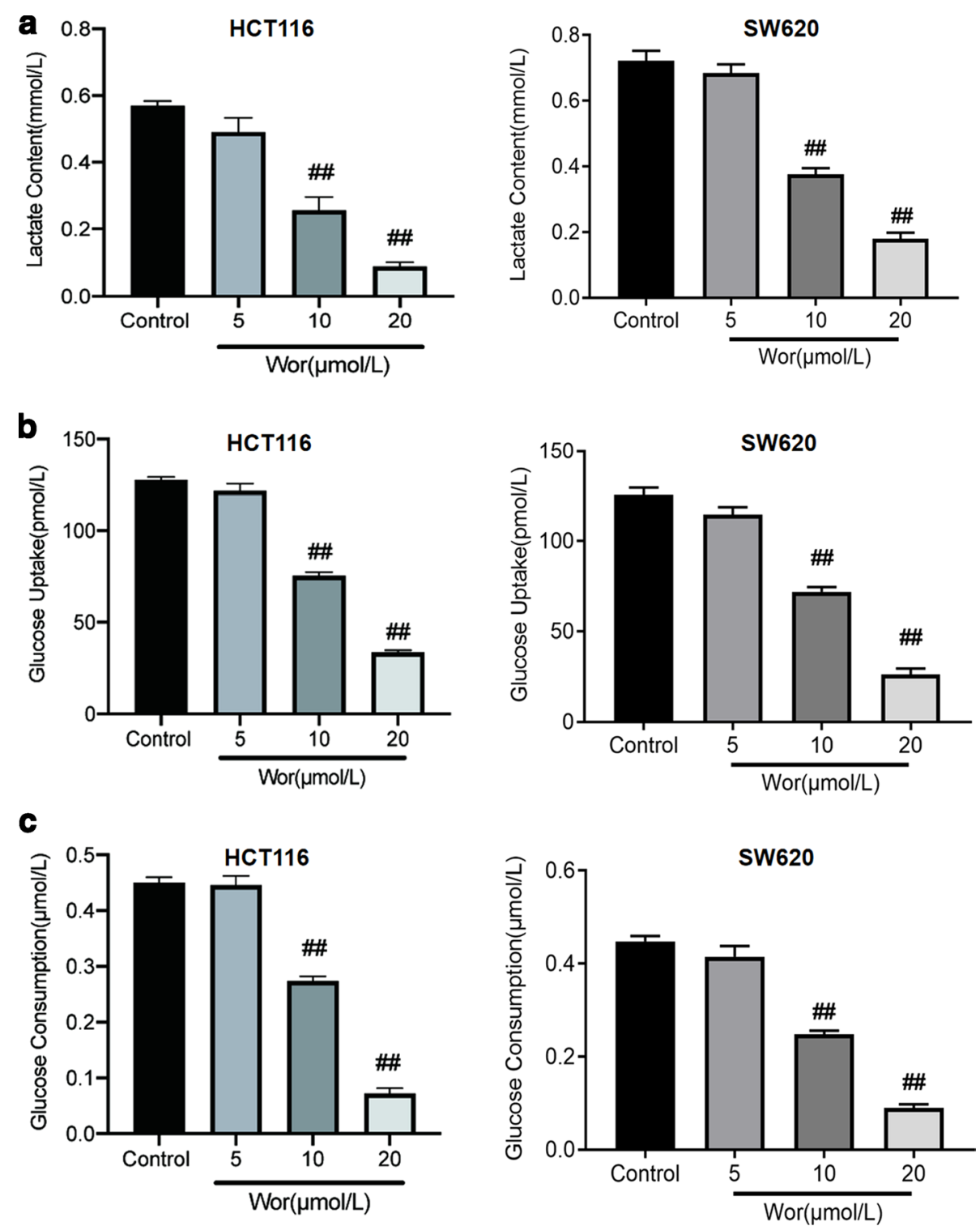

Fig. 2 Glucose consumption, glucose uptake and lactate release for colorectal cancer cells after worenine treatment. a The lactate level in the supernatant was determined using a lactic acid production detection kit. b Glucose uptake was evaluated using a glucose uptake colorimetric assay kit. c Glucose consumption was evaluated using a glucose assay kit. The data are means \pm SEM. ${ }^{\#} \mathrm{p}<0.05,{ }^{\# \#} \mathrm{p}<0.01 \mathrm{vs}$. control group

reduction in the inhibition effect of its product, G-6-P. The activity of PFK remained unchanged. These results suggest that worenine affects glycolysis via the regulation of key glycolytic enzymes.

\section{Worenine promotes HIF-1a degradation}

It is widely known that HIF-1 $\alpha$ is overexpressed in colorectal cancer cells and could increase the activity of glycolytic genes, such as GLUT, HK2 and PKM-2, thus evoking glycolysis. Western blot results of nuclear and membrane fractions of HCT116 cells revealed that worenine inhibited nuclear HIF-1 $\alpha$ more than the membrane HIF- $1 \alpha$ 
a
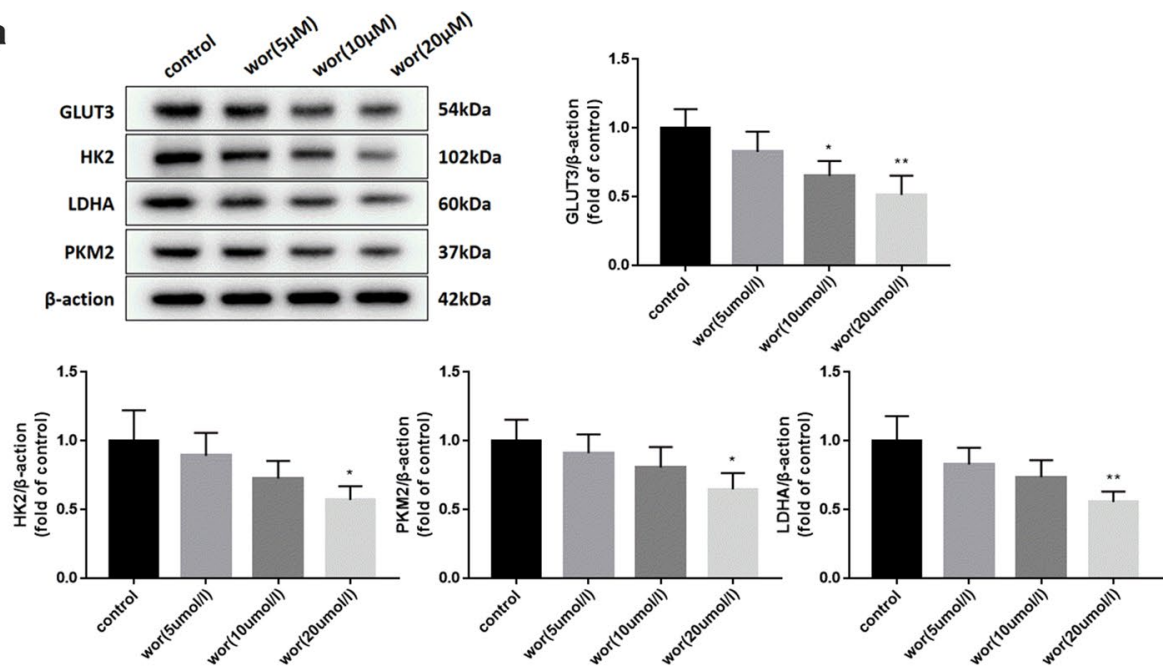

b
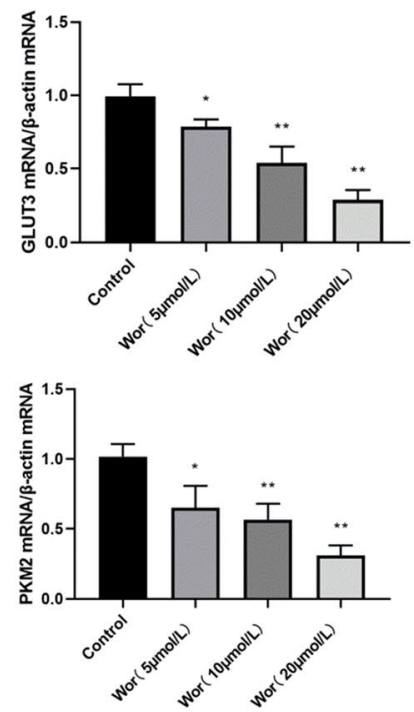

C

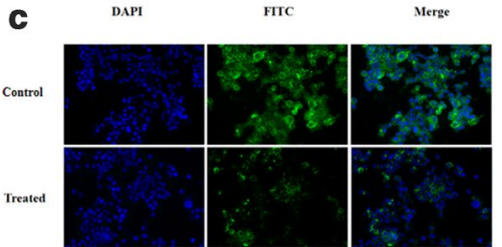

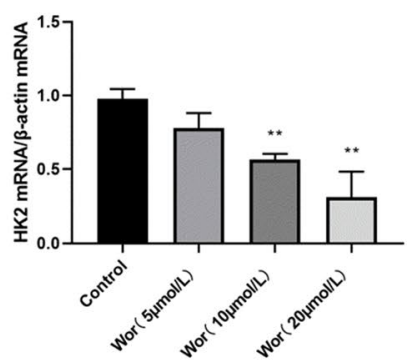

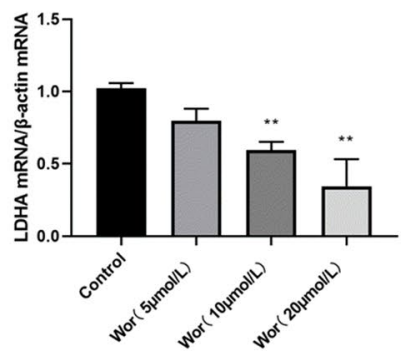

DAP

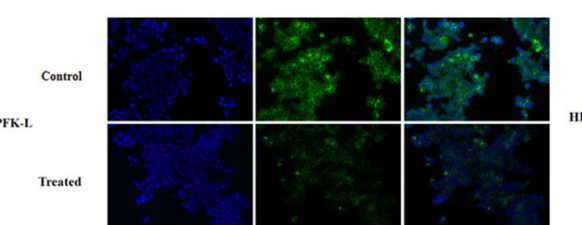

Fig. 3 Key glycolytic enzymes are inhibited by worenine. HCT116 cells were treated with 5, 10 or $20 \mu \mathrm{M}$ worenine for $24 \mathrm{~h}$. a The protein expressions of GLUT3, HK2, PKM2 and LDHA were determined using western blotting. $\mathbf{b}$ he results for the quantitative RT-PCR analysis of the mRNA expressions of GLUT3, HK2, PKM2 and LDHA in worenine-treated cells. $\mathbf{c}$ HK-2 and PFK-1 expression were evaluated using immunofluorescence. Cells were co-stained with DAPI to visualize the nuclei. The data are means \pm SEM. ${ }^{\#} \mathrm{p}<0.05$, ${ }^{\# \#} \mathrm{p}<0.01 \mathrm{vs}$. control group

(Fig. 4a and b). Further results (Fig. 4c) suggest that Worenine elevated pVHL levels and decreased HIF- $1 \alpha$ protein expression after treatment with the protein synthesis inhibitor. The fluorescent images showed similar results (Fig. 4d). The fluorescent puncta of HIF- $1 \alpha$ was weakened by worenine. Based on these results, we conjecture 


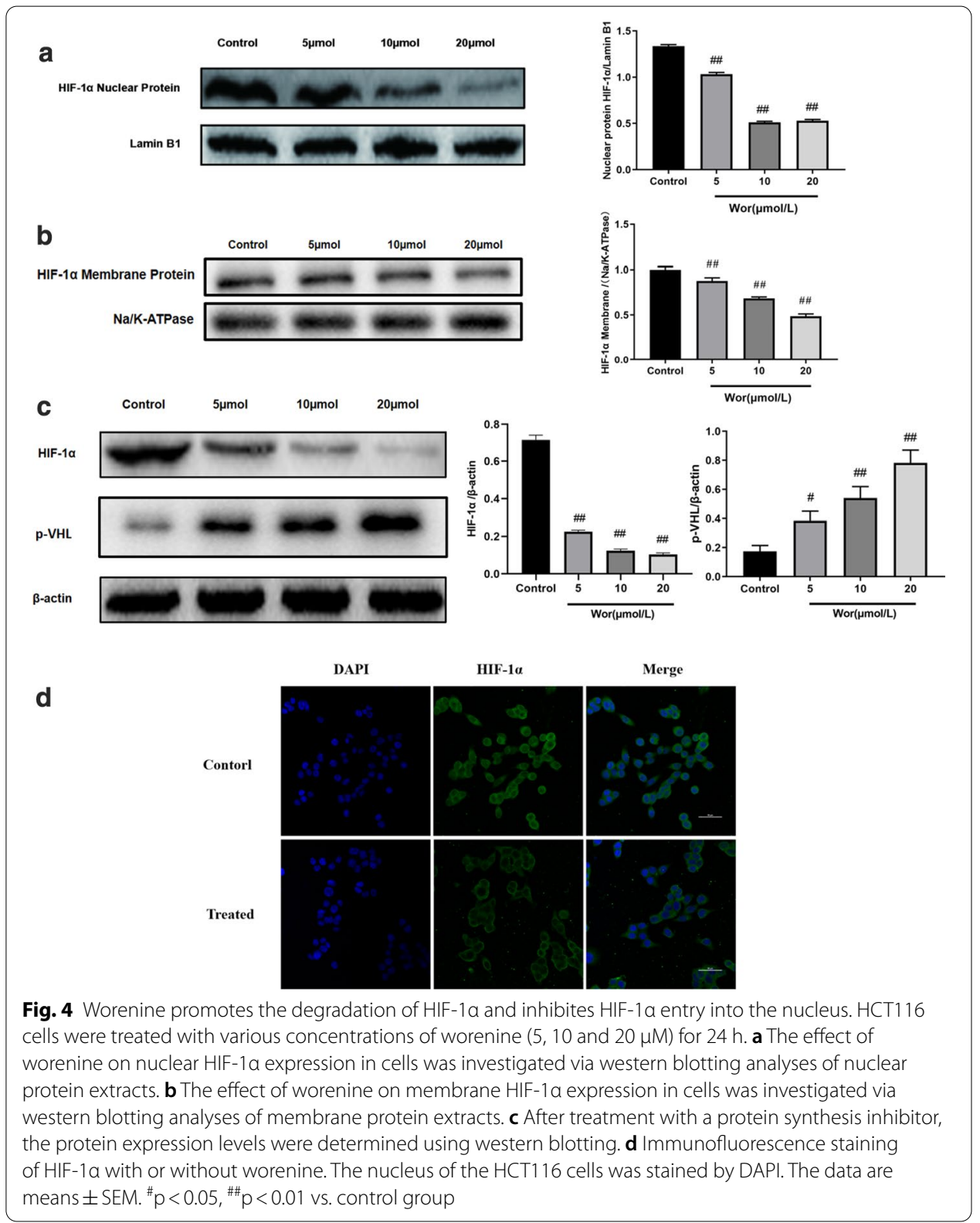

that Worenine could decrease the HIF- $1 \alpha$ level in HCT 116 cells by preventing HIF- $1 \alpha$ nuclear translocation and promoting HIF- $1 \alpha$ degradation.

\section{Worenine exerts an inhibitory effect on glycolysis and cell viability through HIF-1a signaling}

To determine the role of HIF- $1 \alpha$ in worenine-induced effects on the glycolysis and cell growth of HCT116 cells, HIF- $1 \alpha$ siRNA (siHIF-1 $\alpha$ ) and desferrioxamine (Dfx) were respectively used to inhibit and stabilize HIF- $1 \alpha$. The expression of HIF- $1 \alpha$ protein was knocked down by siHIF-1 $\alpha$ (Fig. 5). Similarly to worenine treatment, siHIF-1 $\alpha$ downregulated the mRNA and protein levels of HK-2 and PKM2. Desferrioxamine (Dfx) is an iron chelator that can stabilize HIF-1 $\alpha$ without altering HIF-1 $\alpha$ mRNA expression. 


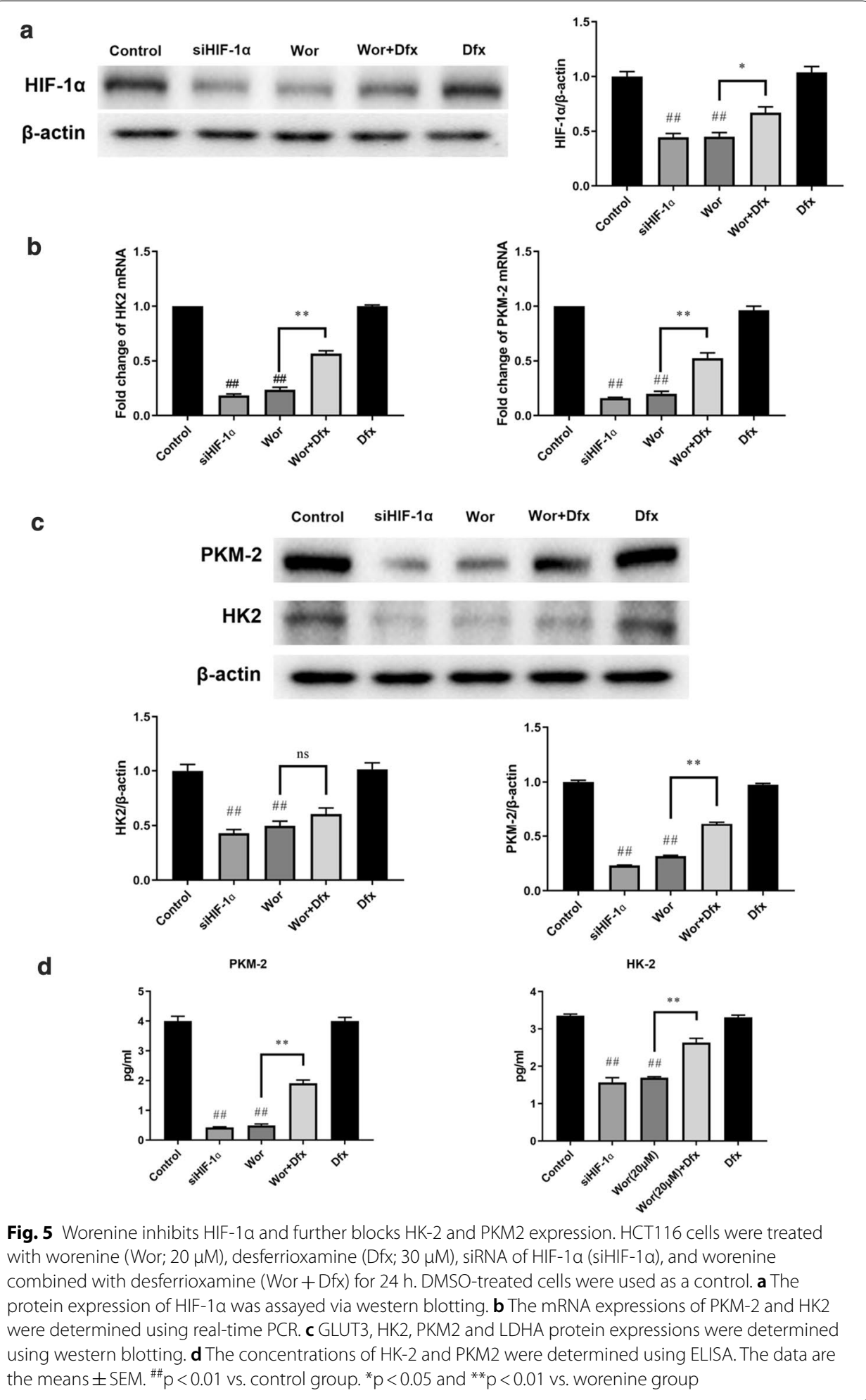

With the stabilization of HIF- $1 \alpha$ by Dfx, the decrease in HK-2 and PKM2 expression induced by worenine treatment was reversed. In addition, Dfx $(30 \mu \mathrm{M})$ alone did not affect HIF-1 $\alpha$, HK-2 and PKM2 expression. 
The effects of worenine on glucose uptake, glucose consumption and lactate generation were mimicked by siHIF- $1 \alpha$ and weakened by Dfx treatment (Additional file 4: Figure S4A-C). Furthermore, a remarkable decrease in cell viability was observed after treatment with $20 \mu \mathrm{M}$ worenine or siHIF-1 $\alpha$, compared with control group (Additional file 4: Figure S4D). Nevertheless, Dfx treatment hindered the worenine-induced decrease in cell viability, but Dfx $(30 \mu \mathrm{M})$ alone did not affect cell viability and glycolysis. We concluded that worenine inhibits the Warburg effect and cell growth of HCT116 cells by decreasing the levels of HIF- $1 \alpha$.

\section{Discussion}

Worenine isolated from Coptis chinensis was discovered to exhibit anti-cancer activity in HCT116 and SW620 cells in our preliminary MTT-based evaluations. However, the effects and mechanisms of worenine against cancer have not been fully elucidated. Here, we found that worenine inhibited colorectal cancer cell growth, proliferation, cell cycle progression and the Warburg effect by targeting HIF-1 $\alpha$ in vitro.

The Warburg effect refers to cancer cells prioritizing glycolysis rather than oxidative phosphorylation as the means of metabolizing glucose, even in the presence of oxygen, producing lactate as the final product. This metabolic transition helps increase tumor cell proliferation because aerobic glycolysis produces enough energy from limited resources for cancer cells to survive and enable metabolic intermediates to shift from energy production to bio-synthesis [12]. This occurs because the expressions of different glycolytic enzyme isoforms can be changed in cancer cells by certain transcriptional factors, such as HIF- $1 \alpha$.

In colorectal cancer, tumorigenic cells upregulate GLUT3, HK2, PFK-L, PKM2 and LDHA expression, enhancing glycolytic flux and increasing glucose uptake [13]. GLUT1 is the most widely overexpressed transporter in cancer cells and it has been used as an indicator for poor colorectal cancer prognosis [14]. The cancer-specific isoform PKM2 confers tumorigenic cells with the ability to withstand oxidative stress. PFK- 1 is a 380 KDa homo- or hetero-tetramer found in all tissues. It consists of three isoforms: PFK-L, PFK-P and PFK-M. However, HIF-1 $\alpha$ only increases the expression of the PFK-L isoform [15]. HK2 is one of the rate-limiting enzymes involved in the step of producing glucose6-phosphate [16]. It plays an essential role in the regulation of glycolytic flux. A high level of LDHA correlates with aggressive forms of several different tumor types, including colorectal cancer [17]. These molecules, which are involved in glycolysis, can be ideal therapeutic targets for hypoxic and glycolytic tumors [15].

Interestingly, worenine induced downregulations of PFK-L, HK2 and PKM2, indicating that the level of glycolysis, cellular energy production and macromolecular biosynthesis were declining in the tumor cells. In other words, the Warburg effect was reversed by worenine to a certain extent.

In addition, the high level of glycolytic enzymes widely observed in cancer cells has been proven to be essential for tumor cells to grow [18]. These levels are closely associated with poor overall survival in cancer patients. We found that glucose uptake and consumption as well as lactate production in HCT116 and SW620 cells were reduced in a dose-dependent manner after worenine treatment. 
Hypoxia is a feature of solid tumors, including colorectal cancer. It regulates the cancer cell metabolism reprogramming that promotes tumor cell growth, invasion and infiltration. HIF-1 is a major transcription factor under hypoxia. It mediates glycolysis by regulating the expression of glycolytic genes [18]. It is a basic helix-loop-helix heterodimeric transcription factor composed of alpha $(\alpha)$ and beta $(\beta)$ subunits $[19,20]$. HIF- $1 \alpha$ expression persists in cells under normoxia, but it is unstable as it is degraded through the ubiquitin-proteasome (26S) pathway. The ubiquitin E3 ligase complex mediates this degradation, where the von Hippel-Lindau tumor suppressor protein (pVHL) can associate with oxygen-dependent destruction domain(s) on the subunit. However, hypoxia prevents hydroxylation, thus preventing the tumor-suppresive protein from binding to proline on HIF-1 $\alpha$. Consequently, pVHL is inactivated, which prevents HIF- $1 \alpha$ from becoming a target for $26 \mathrm{~S}$ proteasome degradation [21]. This allows HIF-1 $\alpha$ to bind to its corresponding HIF- $1 \beta$ subunit to form the heterodimer HIF-1 transcription factor complex. Then, this complex enters the nucleus and binds to the hypoxia response elements (HREs) on specific strands of DNA, in order to target genes that code for supporting tumor cell survival and increasing proliferative capacity and metastasis potential [22, 23].

Here, worenine decreased the HIF- $1 \alpha$ level by activating p-VHL. Recent studies have suggested that HIF- $1 \alpha$ is a reasonable therapeutic target for cancer treatment [24]. HIF- $1 \alpha$ inhibition by RNA interference or inhibitors could reduce tumor cells growth and metastasis [25, 26]. We found that worenine improved the glycolysis in colorectal cancer cells in a manner similar to HIF-1 $\alpha$ siRNA.

Based on this evidence, we hypothesize that worenine exerts anti-colorectal cancer activity by targeting HIF-1 $\alpha$. To verify our hypothesis, desferrioxamine (Dfx) was used to stimulate HIF- $1 \alpha$ protein aggregation. It is an iron chelator that increases intracellular HIF- $1 \alpha$ levels as the upregulation of HIF prolyl hydroxylases (HIF-PH) requires iron [27]. Dfx treatment reversed worenine-induced effects on the Warburg effect, confirming worenine-induced colorectal cancer cell death to have occurred via HIF-1 $\alpha$ inhibition (Additional file 5).

However, since four aspects are involved in the inhibition of HIF- $1 \alpha$ activity, including protein synthesis, HIF-1 $\alpha$ degradation, transcriptional activity and mRNA levels, further investigation is required to reveal more detailed mechanisms underlying the anti-cancer properties of worenine $[28,29]$. In addition, to sufficiently evaluate whether worenine can be a candidate for the clinical treatment of colorectal cancer, its protective effect should be further confirmed in vivo.

\section{Conclusion}

This study showed that worenine derived from Coptis chinensis exerts anti-colorectal cancer function by affecting the HIF-1 $\alpha$-mediated glycolysis in the cells. Worenineinduced downregulation of HIF-1 $\alpha$ was involved in this process. Our research provides new insights into the development of natural compounds as anti-colorectal cancer drugs.

Abbreviations

HIF-1: Hypoxia-inducible factor-1; PFK: Phosphofructokinase; HK: Hexokinase; PKM2: Pyruvate kinase M2; Dfx: Desferrioxamine; pVHL: Von Hippel-Lindau tumour suppressor protein. 


\section{Supplementary Information}

The online version contains supplementary material available at https://doi.org/10.1186/s11658-021-00263-y.

Additional file 1: Fig. S1. Worenine inhibits cell proliferation and impairscell cycle progression of SW620. SW620 cells were treated with worenine $(0 \sim 20 \mu \mathrm{M})$ as indicated for $24 \mathrm{~h}$. A - Colony formation assay results for SW620. B Cell cycle profile of SW620tested using flow cytometry. $(n=3)$

Additional file 2: Fig. S2. The effect of worenine on FHC glycolysis. FHC cells were treatedwith worenine $(0 \sim 20 \mu \mathrm{M})$ as indicated for 24h. A - The lactate level in the supernatants was determined usinga lactic acid production detection kit. B - Glucose uptake wasevaluated using a glucose uptake colorimetric assay kit. C - Glucose consumptionwas evaluated using a glucose assay kit. The data are means \pm SEM. ${ }^{\#} \mathrm{p}<0.05$, $\# \#<0.01$ vs. control group.

Additional file 3: Fig. S3 The effect of Worenine on glycolysis enzymes.HCT116 cells were treatedwith worenine $(0 \sim 20 \mu \mathrm{M})$ as indicated for $24 \mathrm{~h}$. A - The effect of worenine treatment at different concentrationson the protein expression of PFK-L in cell lysates was determined using a westernblotting assay. B - The effect of worenine treatmentat different concentrations on the protein expression of PFK-L and HK2 in cell supernatants was determined using ELISA. C - The effect of worenine treatment at different concentrationson the activity of PFK, HK and PKM was tested with a phosphofructokinase assaykit, hexokinase assay kit and pyruvate kinase assay kit, respectively. The data are means \pm SEM. ${ }^{\#} \mathrm{p}<0.05,{ }^{\# \#} \mathrm{p}<0.01,{ }^{*} \mathrm{p}<0.05,{ }^{* *} \mathrm{p}<0.01$ vs. control group.

Additional file 4: Fig. S4 Worenine inhibits glycolysisand cell viability by suppressing HIF-1a. HCT116 cells were treated with worenine $(20 \mu \mathrm{M})$, desferrioxamine $(30 \mu \mathrm{M})$, siRNA of HIF-1a, and worenine combinedwith desferrioxamine. DMSO-treated cells were used as a control. The lactate level(A), the glucose uptake level (B) and the glucose consumption level (C) were measuredwith the appropriate kits. D - The cell viability of HCT116 cells was determinedusing an MTT assay. The data are means \pm SEM. ${ }^{\# \#} \mathrm{p}<0.01$ vs. controlgroup. ${ }^{* *} \mathrm{p}<0.01 \mathrm{vs}$. worenine group.

Additional file 5: Original data.

\section{Acknowledgements}

We are indebted to Dr. Tianya Liu of the School of Integrated Chinese and Western Medicine, Nanjing University of Chinese Medicine for providing the drug monomer.

\section{Authors' contributions}

Concept, HC and DS; methodology, L; software, FZ, LL and LW; formal analysis, LJ; investigation, WS; resources, HC; data curation, JQ; writing (original draft preparation), LW; writing (review and editing), JJ and JT; visualization, JT; supervision, DS; project administration, JQ and LT; funding acquisition, YC, HC and DS. All authors have read and agreed to the published version of the manuscript.

\section{Funding}

This research was funded by the National Natural Science Foundation of China (Grant Nos. 82074318, 81930117, 81973523); the National Key R\&D Program of China (Grant No. 2017YFC1700602); and the Priority Academic Program Development of Jiangsu Higher Education Institutions: Changshu Health and Family Planning Commission Supporting Project (Grant Number: CSWS201514); Suzhou Municipal Science and Technology Bureau Supporting Project (Grant Numbers: SYSD2016015, SYSD2019004 and SYSD2019195); Changshu Municipal Science and Technology Bureau Supporting Project (Grant Numbers: CS201623 and CS201808).

\section{Availability of data and materials}

All data from this study are available in this published article.

\section{Declarations}

Ethics approval and consent to participate

Not applicable.

\section{Consent for publication}

Not applicable.

\section{Competing interests}

The authors declare that they have no competing interests.

\section{Author details}

${ }^{1}$ Changshu TCM Hospital Affiliated to Nanjing University of Chinese Medicine, Changshu 215500, China. ${ }^{2}$ Collaborative Innovation Center of Jiangsu Province of Cancer Prevention and Treatment of Chinese Medicine, Nanjing 210023, China. ${ }^{3}$ School of Integrated Chinese and Western Medicine, Nanjing University of Chinese Medicine, Nanjing 210023, China. ${ }^{4}$ The First School of Clinical Medicine, The Affiliated Hospital of Nanjing University of Chinese Medicine, Nanjing 210029, China.

Received: 10 November 2020 Accepted: 10 May 2021

Published online: 18 May 2021 
References

1. Amin M, Lockhart AC. The potential role of immunotherapy to treat colorectal cancer. Expert Opin Inv Drug. 2015;24:329-44.

2. Connell LC, Mota JM, Braghiroli MI, Hoff PM. The rising incidence of younger patients with colorectal cancer: questions about screening, biology, and treatment. Curr Treat Options Oncol. 2017;18:23.

3. Wang Q, Shi YL, Zhou K, Wang LL, Yan ZX, Liu YL, et al. PIK3CA mutations confer resistance to first-line chemotherapy in colorectal cancer. Cell Death Dis. 2018;9:739.

4. Meng FC, Wu ZF, Yin ZQ, Lin LG, Wang R, Zhang QW. Coptidis rhizoma and its main bioactive components: recent advances in chemical investigation, quality evaluation and pharmacological activity. Chin Med. 2018;13:13.

5. Kang JX, Liu J, Wang J, He C, Li FP. The extract of huanglian, a medicinal herb, induces cell growth arrest and apoptosis by upregulation of interferon-beta and TNF-alpha in human breast cancer cells. Carcinogenesis. 2005:26:1934-9.

6. Jiang X, Huang LF, Wu LB, Wang ZH, Chen SL. UPLC-QTOF/MS analysis of alkaloids in traditional processed Coptis chinensis Franch. Evid Based Compl Alt. 2012;5:942384.

7. Huang T, Xiao Y, Yi L, Li L, Wang M, Tian C, et al. Coptisine from Rhizoma coptidis suppresses HCT-116 cells-related tumor growth in vitro and in vivo. Sci Rep. 2017;7:38524.

8. Liu L, Wang ZB, Song Y, Yang J, Wu LJ, Yang BY, et al. Simultaneous determination of eight alkaloids in rat plasma by UHPLC-MS/MS after oral administration of Coptis deltoidea C. Y. Cheng et Hsiao and Coptis chinensis Franch. Molecules. 2016;21:913.

9. Matthew GVH, Lewis CC, Craig BT. Understanding the Warburg effect: the metabolic requirements of cell proliferation. Science. 2009;324:1029-33.

10. Semenza GL. Hypoxia-inducible factors: coupling glucose metabolism and redox regulation with induction of the breast cancer stem cell phenotype. EMBO J. 2017:36:252-9.

11. Dongdong S, Feng Z, Jie Q, Weixing S, Huisen F, Jiani T, et al. 4'-hydroxywogonin inhibits colorectal cancer angiogenesis by disrupting PI3K/AKT signaling. Chem-Biol Interact. 2018;296:26-33.

12. Gwangwa MV, Joubert AM, Visagie MH. Crosstalk between the Warburg effect, redox regulation and autophagy induction in tumourigenesis. Cell Mol Biol Lett. 2018;23:20.

13. Marın-Hernandez A, Rodrıguez-Enrıquez S, Vital-Gonzalez PA, FloresRodrıguez FL, Macıas-Silva M, Sosa-Garrocho M, et al. Determining and understanding the control of glycolysis in fast-growth tumor cells. Flux control by an over-expressed but strongly product-inhibited hexokinase. Febs J. 2006;273:1975-88.

14. Macheda ML, Rogers S, Best JD. Molecular and cellular regulation of glucose transporter (GLUT) proteins in cancer. J Cell Physiol. 2005;202:654-62.

15. Marin-Hernandez A, Gallardo-Perez JC, Ralph SJ, Rodriguez-Enriquez S, Moreno-Sanchez R. HIF-1a modulates energy metabolism in cancer cells by inducing over-expression of specific glycolytic isoforms. Mini-Rev Med Chem. 2009;9:1084-101.

16. Patra KH, Wang Q, Bhaskar P, Miller L, Wang Z, Wheaton W, et al. Hexokinase 2 is required for tumor initiation and maintenance and its systemic deletion is therapeutic in mouse models of cancer. Cancer Cell. 2013;24:213-28.

17. Koukourakis MI, Giatromanolaki A, Simopoulos C, Polychronidis A, Sivridis E. Lactate dehydrogenase 5 (LDH5) relates to up-regulated hypoxia inducible factor pathway and metastasis in colorectal cancer. Clin Exp Metastasis. 2005;22:25-30.

18. Wang H, Wang L, Zhang Y, Wang J, Lin D. Inhibition of glycolytic enzyme hexokinase II (HK2) suppresses lung tumor growth. Cancer Cell Int. 2016;16:9.

19. Courtnay R, Ngo DC, Malik N, Ververis K, Tortorella SM, Karagiannis TC. Cancer metabolism and the Warburg effect: the role of HIF-1 and PI3K. Mol Biol Rep. 2015:42:841-51.

20. Wang GL, Jiang BH, Rue EA, Semenza GL. Hypoxia-inducible factor 1 is a basic-helix-loop-helix-PAS heterodimer regulated by cellular O2 tension. PNAS. 1995:92:5510-4.

21. Ji AK, Da KC, Min JS, Kang I, Jin CK, Kim S, et al. VBP1 represses cancer metastasis by enhancing HIF-1 a degradation induced by pVHL. Febs J. 2018:285:115-26.

22. Lisy K, Peet DJ. Turn me on: regulating HIF transcriptional activity. Cell Death Differ. 2008;15:642-9.

23. Momin S, Nagaraju GP. The role of hypoxia-inducible factor 1-alpha in colorectal cancer. theranostics approaches to gastric and colon cancer. 2020; pp. 61-68

24. Feng W, Tao X, Huang S, Shi Q, Tang C, Ge C, et al. HIF-1 a promotes the migration and invasion of hepatocellular carcinoma cells via the IL-8-NF-kB axis. Cell MOL Biol Lett. 2018;23:26.

25. Choi HJ, Eun JS, Kim BG, Kim SY, Soh Y. Vitexin, an HIF-1a inhibitor, has anti-metastatic potential in PC12 cells. Mol Cells. 2007;22:291-9.

26. Takahashi Y, Nishikawa M, Takakura Y. Inhibition of tumor cell growth in the liver by RNA interference-mediated suppression of HIF-1 a expression in tumor cells and hepatocytes. Gene Ther. 2008:15:572-82.

27. Woo K, Lee T, et al. Desferrioxamine, an iron chelator, enhances HIF-1a accumulation via cyclooxygenase-2 signaling pathway. Biochem Biophys Res Commun. 2006;343:8-14

28. Bullen JW, Tchernyshyov I, Holewinski RJ, Devine L, Wu F, Venkatraman V, et al. Protein kinase A-dependent phosphorylation stimulates the transcriptional activity of hypoxia-inducible factor 1. Sci Signal. 2016;9:ra56.

29. Klaus A, Fathi O, Tatjana T-W, et al. Expression of hypoxia-associated protein HIF-1 alpha in follicular thyroid cancer is associated with distant metastasis. Pathol Oncol Res. 2018;24:289-96.

\section{Publisher's Note}

Springer Nature remains neutral with regard to jurisdictional claims in published maps and institutional affiliations. 